\title{
The characterisation of interstitial lung disease multidisciplinary team meetings: a global study
}

\author{
Luca Richeldi ${ }^{1}$, Naomi Launders ${ }^{2}$, Fernando Martinez ${ }^{3}$, Simon L.F. Walsh ${ }^{4}$, \\ Jeffrey Myers ${ }^{5}$, Bonnie Wang ${ }^{6}$, Mark Jones ${ }^{7}$, Alison Chisholm ${ }^{2}$ and \\ Kevin R. Flaherty ${ }^{6}$ on behalf of the Respiratory Effectiveness Group IPF/ILD \\ Working Group ${ }^{8}$
}

Affiliations: ${ }^{1}$ Unità Operativa Complessa di Pneumologia, Fondazione Policlinico Universitario A. Gemelli IRCCS, Università Cattolica del Sacro Cuore, Rome, Italy. ${ }^{2}$ Respiratory Effectiveness Group, Cambridge, UK. ${ }^{3}$ Joan and Sanford I. Weill Dept of Medicine, New York-Presbyterian Hospital/Weill Cornell Medical Center, New York, NY, USA. ${ }^{4}$ Dept of Radiology, Royal Brompton Hospital, London, UK. ${ }^{5}$ Dept of Pathology, University of Michigan, Ann Arbor, MI, USA. 'Division of Pulmonary and Critical Care Medicine, Dept of Medicine, University of Michigan, Ann Arbor, MI, USA. ${ }^{7}$ NIHR Southampton Biomedical Research Centre, Clinical and Experimental Sciences, Faculty of Medicine, University of Southampton, Southampton, UK. ${ }^{8}$ A list of the Respiratory Effectiveness Group IPF/ILD Working Group collaborators can be found in the Acknowledgements section.

Correspondence: Luca Richeldi, Unità Operativa Complessa di Pneumologia, Fondazione Policlinico Universitario A. Gemelli IRCCS, Università Cattolica del Sacro Cuore, Largo Agostino Gemelli 8, 00168 Rome. E-mail: luca.richeldidpoliclinicogemelli.it

ABSTRACT Multidisciplinary team (MDT) diagnosis of interstitial lung disease (ILD) has been proposed as a gold standard, but there are no formal recommendations for MDT process or composition and limited knowledge regarding prevalence in routine practice.

We performed a systematic evaluation of ILD diagnostic practice across a range of healthcare settings around the world. Electronic questionnaires were distributed across all global regions via society and collaborators networks.

Responses from 457 unique centres across 64 countries were included in the analysis. Of the 350 (76.6\%) centres holding formal meetings, the majority held face-to-face MDT meetings (80\%), for a minimum of $30 \mathrm{~min}$ (93\%), and discussed diagnosis (96.9\%) and patient management (94.9\%) at the meetings. Compared with non-academic and academic non-ILD centres, ILD academic centres reported a higher ILD caseload, held more formal MDT meetings, and were more likely to include histopathology and rheumatology specialists in their diagnostic team. Of the centres holding MDT meetings, 5.5\% routinely discussed all new cases at such meetings.

An MDT approach to ILD diagnosis is consistently interpreted and widely implemented across a range of routine care settings around the world. This observation will inform future ILD diagnostic agreement studies and diagnostic pathway recommendations.

@ERSpublications

In real-world practice, ILD diagnosis uses a multidisciplinary team approach, irrespective of country or healthcare setting http://ow.ly/I1Di30nMNTX

Cite this article as: Richeldi L, Launders N, Martinez F, et al. The characterisation of interstitial lung disease multidisciplinary team meetings: a global study. ERJ Open Res 2019; 5: 00209-2018 [https:// doi.org/10.1183/23120541.00209-2018].

This article has supplementary material available from openres.ersjournals.com.

Received: Dec 072018 | Accepted after revision: Feb 052019

Copyright $\odot$ ERS 2019. This article is open access and distributed under the terms of the Creative Commons Attribution Non-Commercial Licence 4.0. 


\section{Introduction}

Interstitial lung disease (ILD) is a diverse group of diseases with markedly different prognoses and therapeutic options; idiopathic pulmonary fibrosis (IPF) is the most common and lethal [1, 2]. The licensed antifibrotic agents, pirfenidone and nintedanib, can slow the progression of IPF [3], but they do not reverse the disease. Thus, their arrival increases the emphasis on earlier IPF diagnosis and its accurate differentiation from other ILDs to optimise potential therapeutic benefits.

In 2002, the American Thoracic Society (ATS)/European Respiratory Society (ERS) joint statement on the classification of idiopathic interstitial pneumonias [4] recommended a dynamic process of multidisciplinary team (MDT) diagnosis involving clinicians, radiologists and pathologists for diagnosis of ILD. This recommendation was later reiterated in updated statements in 2013 [5] and 2018 [6].

Diagnosis by an MDT has been shown to improve diagnostic confidence $[7,8]$ and is considered by many the gold standard for ILD. In a global study of ILD diagnostic agreement, regular MDT meeting attendance was associated with improved diagnostic reproducibility, bringing the diagnostic performance of experienced non-university practitioners up to the levels achieved by IPF experts [9]. While highly experienced physicians may discriminate between IPF and non-IPF ILD with a degree of accuracy that rivals an MDT [9], the process of a formal MDT meeting may result in a change of diagnosis [10], and allows for knowledge exchange and broadening of expertise.

Despite these benefits of MDT meetings, a 2017 Fleischner Society White Paper [11] suggested that MDT meetings are not required in all cases, while 2017 French guidelines [12] suggest MDT meetings should occur in specialised centres. The French guidelines [12] state MDT meetings should be held in the presence of relevant specialists as well as the attending pulmonologist, while the White Paper [11], and the 2018 ATS/ERS joint statement [6], specify that MDT meetings should include a pulmonologist, radiologist and pathologist.

The ATS/ERS joint statement called for further research into the optimisation of MDTs [6]. Little is known of how fully these guidelines are implemented in real-world practice. Given that there is a lack of evidence regarding the optimal structure of MDT meetings in general, it is not surprising there is variable guidance. Currently, the lack of specific guidelines for MDT meetings creates an area for greatly needed study.

Understanding the current landscape of how MDT meetings are conducted is an important first step in understanding the optimal structure and approach of MDT meetings in the diagnosis of ILDs. The aim of this study was to describe characteristics of current MDT practices around the world and their role in ILD diagnosis, across a wide range of global and healthcare resource settings. Only after we learn about the current state of MDT meetings can we then study their impact on diagnostic accuracy and identify best practices for guideline statements.

\section{Materials and methods \\ Centre participation}

Centres were invited to participate between November 10, 2016 and March 31, 2017. A pragmatic, inclusive approach to participation was used to ensure representation from a wide range of settings. Known collaborators in 20 countries acted as regional points of contact, providing local expertise on the geographical distribution of diagnostic centres and distributing the electronic study questionnaire and invitation e-mail through local consortia, networks and professional links. The questionnaire was also distributed more broadly via three respiratory society e-mail lists (South African Thoracic Society, Pan African Thoracic Society and Lebanese Pulmonary Society) and advertised on the Respiratory Effectiveness Group website (http://effectivenessevaluation.org) to enable volunteer participation of eligible centres. To ensure a broad and inclusive approach, the only eligibility criterion was that respondents had to be personally involved in the diagnosis of ILD at their centres.

\section{Study design}

A standardised, systematic questionnaire was developed using an electronic data capture tool (Qualtrics Research Suite; Qualtrics, Provo, UT, USA). The questionnaire drew on published questionnaires relating to aspects of ILD diagnosis, and was further refined and tailored to the purposes of this study through ILD physician expert consensus $[13,14]$. Conditional logic was built into the questionnaire to minimise the burden on the respondent, to support data validation and to aid in quality assurance.

The questionnaire included both contextual and outcome questions (supplementary file S1). Contextual questions covered: participants' clinical experience; type of centre (academic/non-academic and/or ILD specialist centre); and ILD caseload and distribution of ILD diagnoses. Outcome questions captured details of: diagnostic tests performed before and/or at the respondent centre; key diagnostic meeting characteristics; percentage of new cases presented at formal ILD diagnostic meetings; and access to licensed antifibrotic agents. No definitions were given for "academic centres" or "ILD centres" and 
therefore interpretation is as classified by the respondent. "Formal" meetings were defined as those of a scheduled or pre-planned nature, whereas "informal" meetings were defined as spontaneous and/or unplanned.

\section{Analysis}

Where multiple responses were received from one centre, the responses were combined into a single response. For questions about the participant, the response from the respondent with the greatest experience was taken, while numerical responses were presented as an average of the responses from that centre. Text responses and ranges were combined where possible. Where this was not possible, the most pessimistic response was taken. The economic status of a country was defined using the World Bank Income Groups [15]. Data were analysed using R version 3.3.3 (www.r-project.org). Results are reported as percentages, mean with standard deviation or median (interquartile range (IQR)) and group comparisons made using the Kruskal-Wallis test, Chi-squared test or Fisher's exact test.

\section{Results}

Between November 10, 2016 and March 31, 2017, 1633 centres were invited to take part in the study and further centres recruited indirectly. In total, 570 responses were received. The overall response rate could not be calculated as denominator data are not available for individuals recruited indirectly through study collaborator networks $(n=215)$ or who responded through the website $(n=68)$. The response rate for those recruited through e-mails by study collaborators was $48.4 \%$ (247 out of 510 ), while the response rate for those recruited through pulmonary society e-mail lists (which will have included many non-ILD specialists) was $5 \%$ (40 out of 805 ) (figure 1).

Of the 570 responses collected, seven participants did not complete the questionnaire, 17 were not directly involved in ILD diagnosis, and 89 were identified as duplicate responses and merged with existing records (figure 1).

\section{Centre and respondent demographics}

A total of 457 valid responses from 64 countries were included in the analysis, of which $90.8 \%$ (415) were completed between November 2016 and January 2017. The median (IQR) time taken to complete a questionnaire was 18.5 (11.6-31.7) min. However, as the questionnaire allowed respondents to save and return to their response, this likely overestimates the average questionnaire duration.

The majority $(\mathrm{n}=318(69.6 \%))$ of respondents reported $\geqslant 11$ years experience post-specialisation and 404 (88.4\%) were pulmonology specialists. Europe contributed the greatest number of centres $(\mathrm{n}=173(37.9 \%))$ followed by Asia-Pacific $(n=120(26.3 \%))$, with the lowest number of centres in the Middle East $(n=24$ $(5.3 \%))$ and Africa $(n=16(3.5 \%))$. Most centres were in high-income countries $(n=306(67.0 \%))$, with only

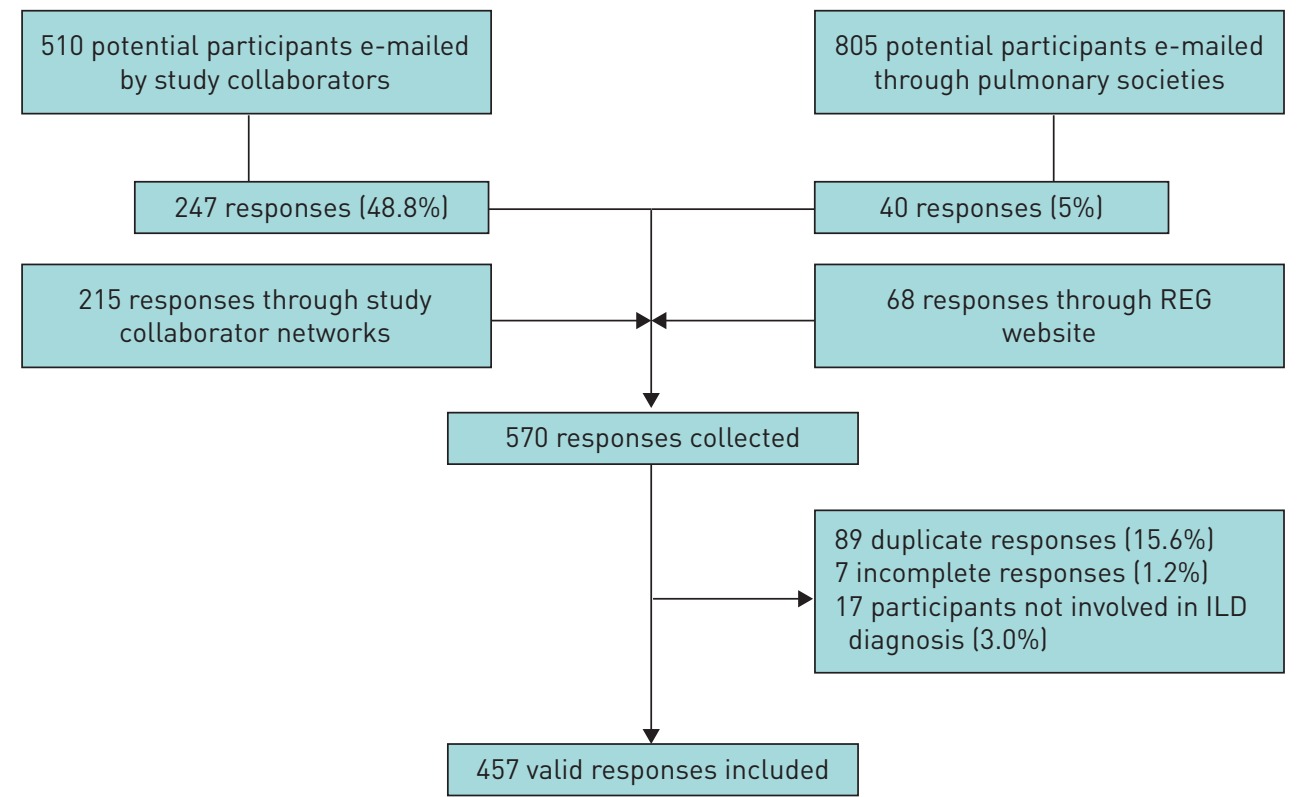

FIGURE 1 Flowchart of participant recruitment. REG: Respiratory Effectiveness Group; ILD: interstitial lung disease. 
two from low-income countries $(0.4 \%)$ (table 1$)$. Nearly half the respondents reported working in ILD academic centres $(n=205(44.9 \%)), 29.1 \%$ in non-ILD academic centres $(n=133)$ and $26.0 \%$ in non-academic centres $(n=119)$.

\section{Caseload, case mix and case management}

Centres saw an estimated median (IQR) 16 (10-28) new ILD cases per month, of which 4 (2-7) were IPF cases. Non-ILD academic centres and non-academic centres reported similar IPF $(p=0.918)$ and non-IPF ILD $(\mathrm{p}=0.252)$ caseloads, while ILD academic centres reported significantly higher caseloads of both IPF and non-IPF ILD than other centre types $(\mathrm{p}<0.001)$ (table 1$)$.

Overall, IPF was the most frequently reported ILD diagnosis and accounted for a mean \pm SD $23.4 \pm 14.5 \%$ of ILD cases; connective tissue disease-related ILD $(16.1 \pm 10.7 \%)$ and sarcoidosis $(15.7 \pm 13.4 \%)$ were the next most common diagnoses. Europe was the only global region where IPF was not the most common ILD diagnosis; European centres reported a higher incidence of sarcoidosis than IPF (20.8\% versus $20.1 \%$, respectively).

ILD academic centres managed a lower percentage of cases than either non-ILD academic centres or non-academic centres (table 1). The difference, however, was not significant for IPF cases $(\mathrm{p}=0.197)$ and only weakly so for non-IPF cases in ILD academic centres versus non-academic centres $(\mathrm{p}=0.040)$. ILD academic centres were more likely to receive patients referred from another pulmonologist than other centre types $(\mathrm{p}<0.001)$ (table 1$)$.

\section{Diagnostic tests}

All 457 centres reported use of spirometry $(n=453(99.1 \%))$ or diffusing capacity of the lung for carbon monoxide (DLCO) $(n=432(94.5 \%))$ to assess most patients, either prior to or on arrival at the centre. All centres also reported use of high-resolution computed tomography (HRCT) for most patients and 450 centres (98.5\%) reported that chest radiography was performed for most patients.

DLCO was most commonly performed in ILD academic centres $(p=0.020)$, in centres in high-income countries $(\mathrm{p}<0.001)$, and in Europe and North America $(\mathrm{p}<0.001)$ (table 2). No significant differences were seen in the proportion of centres conducting spirometry, HRCT or chest radiography across centre

\section{TABLE 1 Key features of participating centres by centre type}

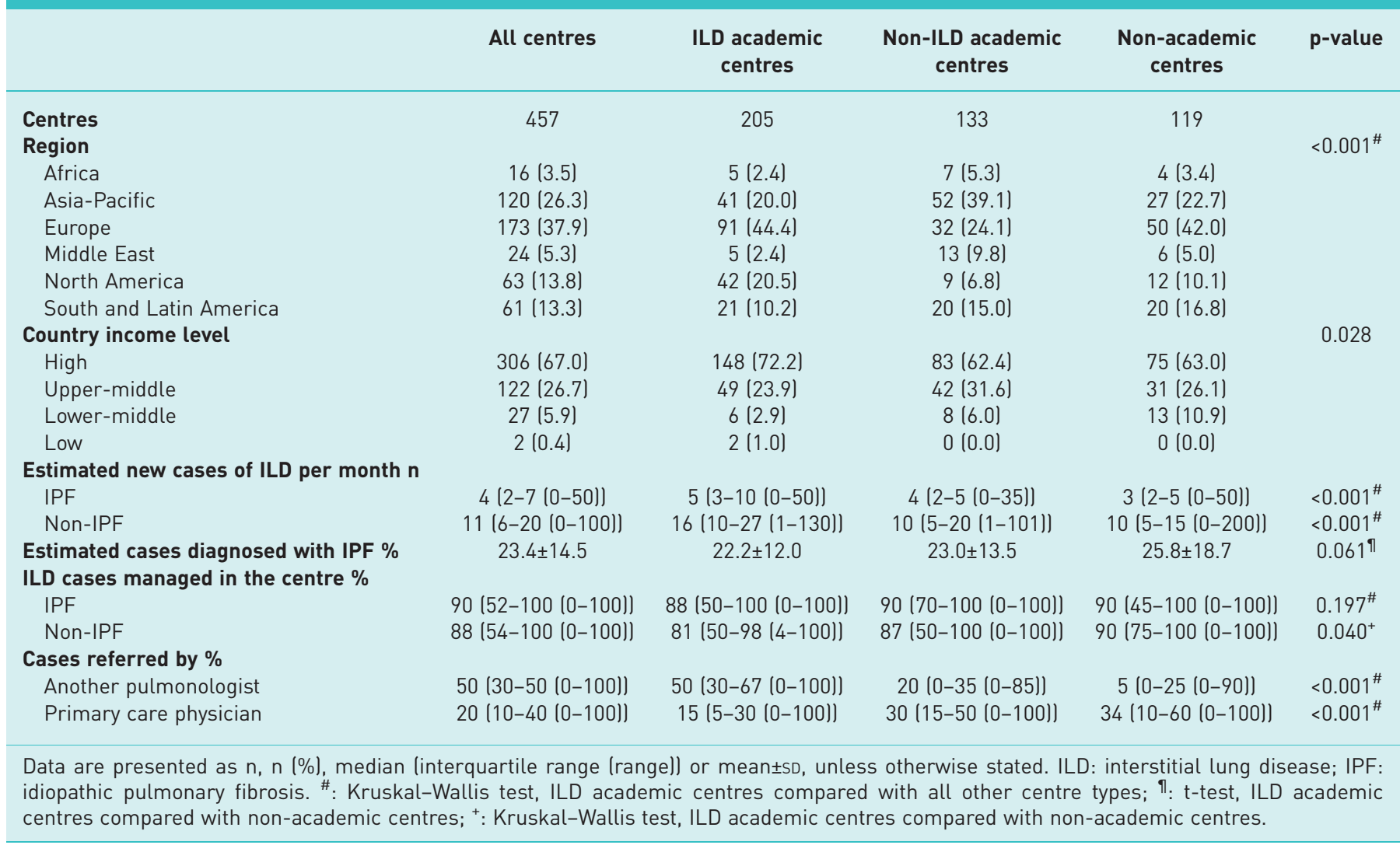


TABLE 2 Diagnostic tests performed in the majority of idiopathic pulmonary fibrosis patients

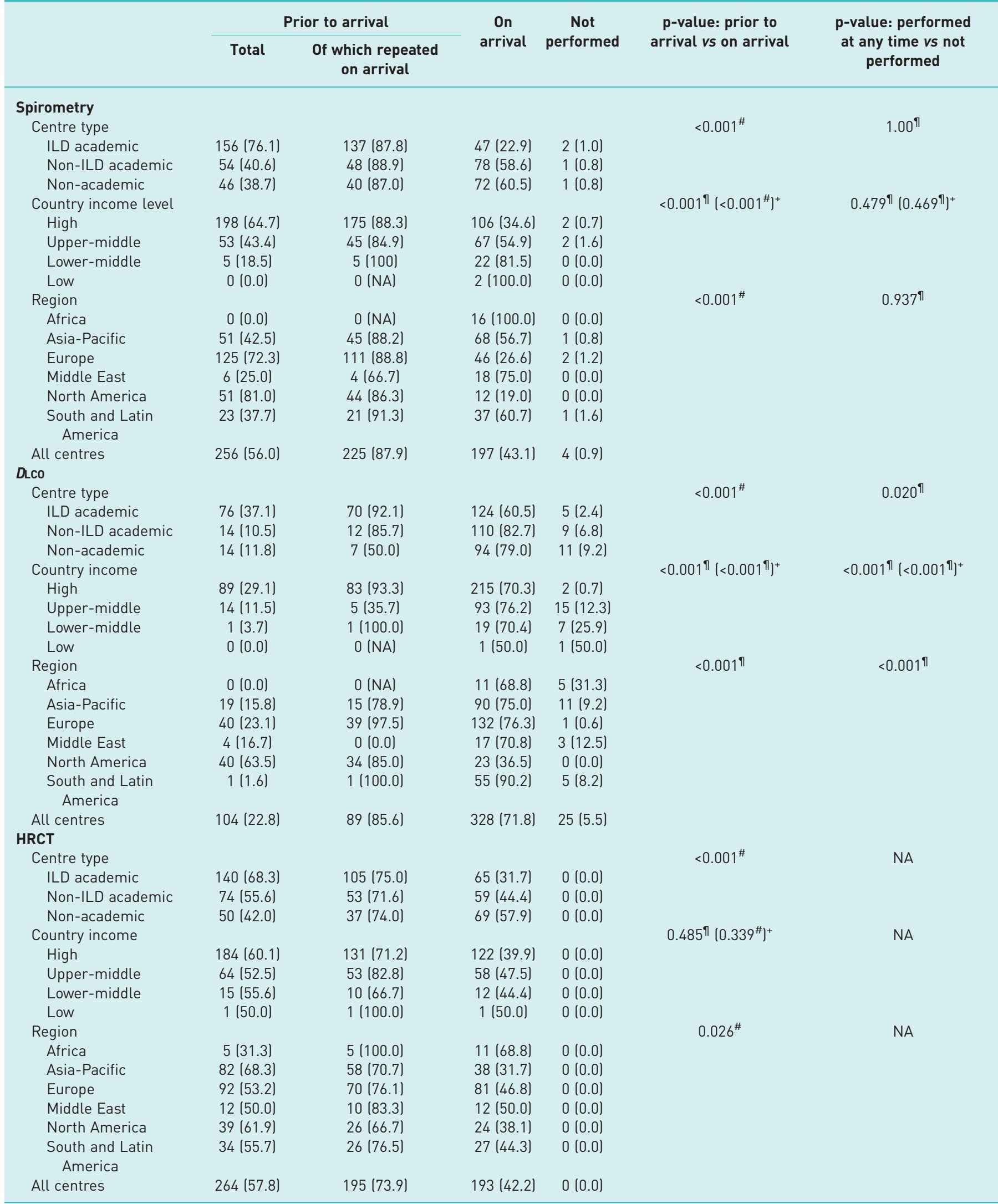




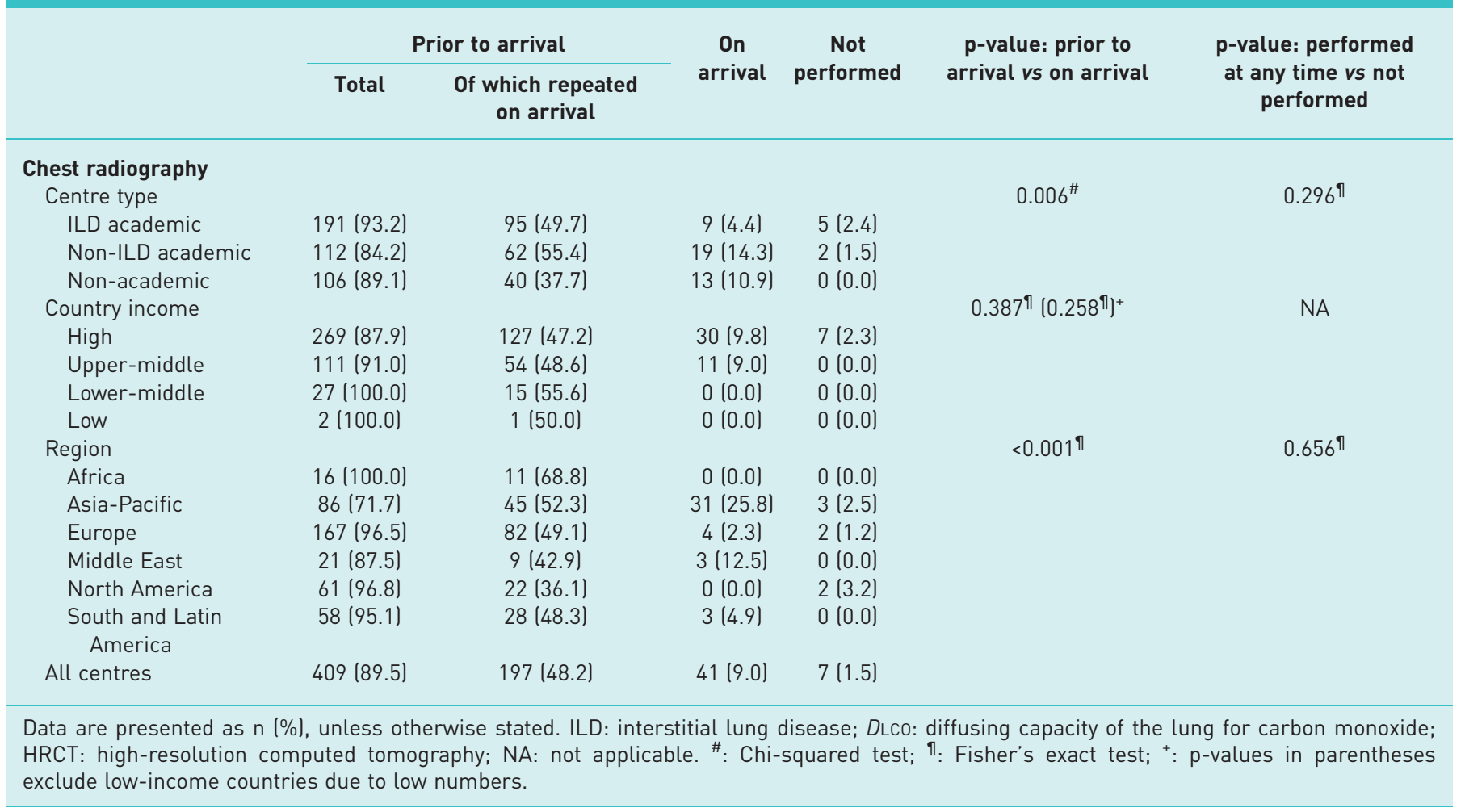

types, country income groups or regions (table 2). Spirometry (56.0\%), HRCT (57.8\%) and chest radiography (89.5\%) were most commonly performed for the first time prior to arrival at the centre, while DLCO was more frequently performed at the centre (71.8\%) (table 2). Most centres repeated spirometry, DLCO and HRCT tests, when these had been performed prior to arrival $(87.9 \%, 85.6 \%$ and $73.9 \%$, respectively). Chest radiography was repeated on arrival by $48.2 \%$ of centres (table 2 ).

Surgical lung biopsy, transbronchial biopsy and bronchoalveolar lavage (BAL) were performed on arrival at the centre in a median (IQR) of $10.0 \%(5.0-25.0 \%), 15.0 \%(5.0-36.0 \%)$ and $50.0 \%(20.0-80.0 \%)$ of patients, respectively (figure 2). Few centres performed endoscopic lung cryobiopsy on arrival $(31.1 \%$ utilised it in at least $1 \%$ of cases). The tests performed on arrival at the centre varied by region, with surgical lung biopsy used most frequently in the Middle East (median (IQR) 20.0\% (10.0-26.3\%) of patients) and South and Latin America (20.0\% (10.0-30.0\%) of patients). Transbronchial biopsy was the most commonly used biopsy technique in the Middle East (40.0\% (12.5-67.8\%) of patients) and BAL in Europe $(80.0 \%(40.0-90.0 \%)$ of patients) (figure 2). Further tests performed are presented in supplementary file S2.

\section{MDT meetings}

Meeting formality

Of the 457 participating centres, 362 (79.2\%) reported holding meetings to discuss new cases and referrals. Of the 95 centres who reported not holding any meetings to discuss cases, 94 (98.9\%) provided a free-text description of the steps they take when they cannot confidently diagnose a case of ILD. Of these, 45 (47.9\%) reported they would discuss the case with colleagues in their centre or at other centres, 35 (37.2\%) that they would consider performing a biopsy and $23(24.5 \%)$ that they may refer the case to another centre.

Physicians in ILD academic centres were more likely to attend meetings (198 out of 205 (96.6\%)) than physicians in non-ILD academic centres (93 out of $133(69.9 \%)$ ) or non-academic centres (71 out of 119 (59.6\%); $\mathrm{p}<0.001)$. Physicians in ILD academic centres also reported attending a higher proportion of formal meetings (median (IQR) 80.5\% (54.8-93.0\%)) than physicians in either non-ILD academic centres $(60.0 \%(31.0-81.0 \%))$ or non-academic centres $(60.0 \%(27.5-90.0 \%) ; \mathrm{p}<0.001)$. Of the 362 physicians attending meetings, $12(3.3 \%)$ reported attending no formal meetings. The median ratio of formal to informal meeting attendance was 3:1. 

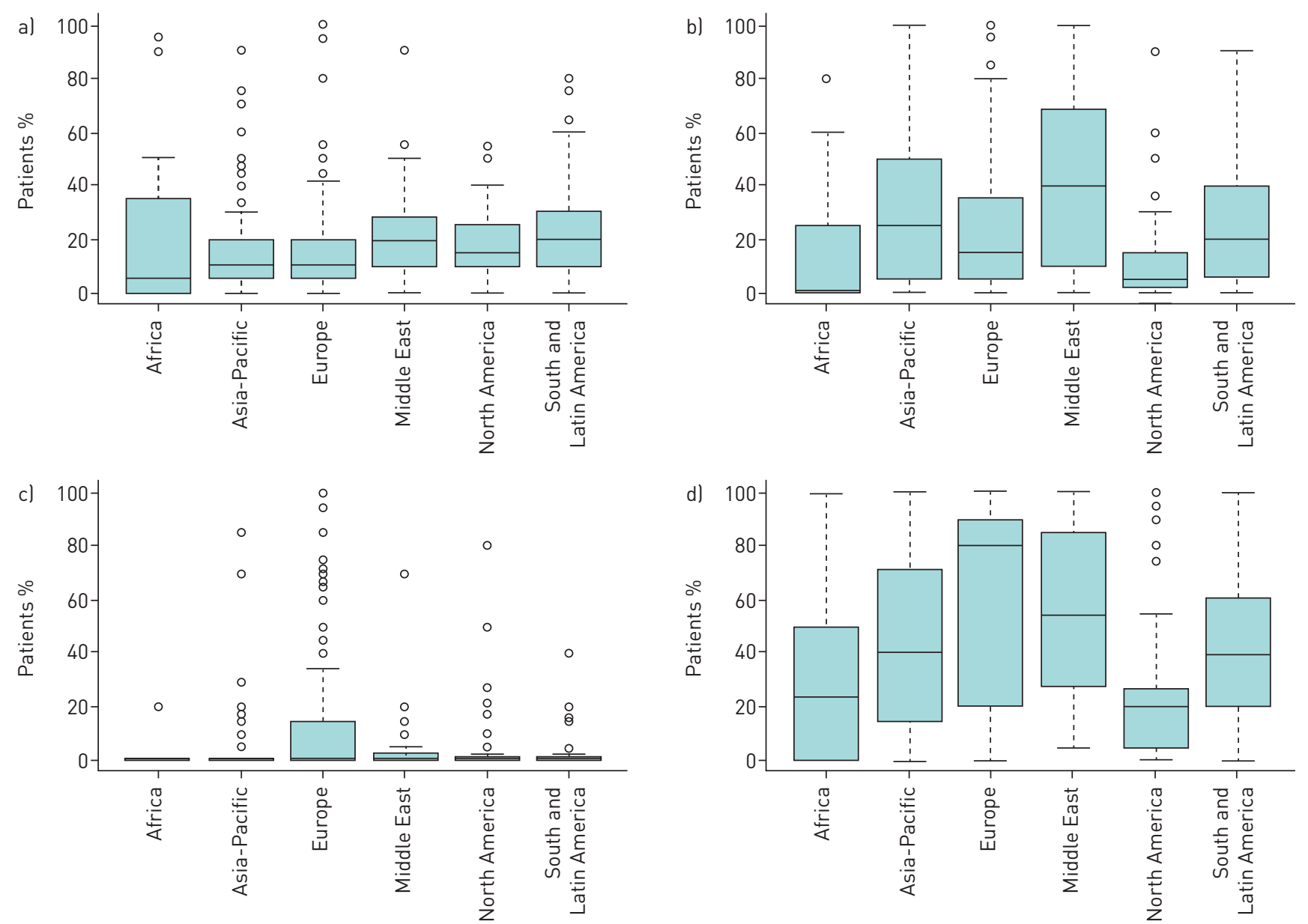

FIGURE 2 Box-and-whisker plots of percentage of patients receiving a) surgical lung biopsy, b) transbronchial biopsy, c) endoscopic lung cryobiopsy or d) bronchoalveolar lavage at the centre by region. The boxes indicate median and interquartile range (IQR), the whiskers indicate 1.5 IQR, and the circles indicate individual outliers.

\section{Case representation}

Centres reported that a median (IQR) of 75\% (50-90\%) of new ILD cases were discussed at formal meetings. 56 centres (15.5\%) reported $100 \%$ of cases were discussed at formal meetings, while 14 centres (3.9\%) reported cases were only presented at informal meetings. There was no significant difference between the median (IQR) percentage of new cases discussed at formal meetings in ILD academic centres (77.5\% (50.0-90.0\%)) compared with non-ILD academic centres $(70.0 \%(40.0-85.0 \%))$ or non-academic centres $(77.0 \%$ (42.0-94.5\%); $\mathrm{p}=0.072)$. However, there was some evidence of regional variation, with centres in Europe reporting they discussed a median (IQR) of $80.0 \%$ (61.0-92.0\%) of cases at formal meetings compared with $64.0 \%(30.0-86.0 \%)$ in South and Latin America and $60.0 \%(45.5-88.0 \%)$ in Africa $(\mathrm{p}=0.049)$. Centres in lower- to middle-income countries reported discussing a lower proportion of new cases in formal meetings (median (IQR) $50.0 \%(20.0-87.0 \%)$ ) than centres in upper-middle- $(67.0 \%$ $(40.0-85.5 \%))$ or high-income countries $(80.0 \%(58.8-92.0 \%) ; \mathrm{p}=0.006)$. Only one of the two centres in low-income countries reported holding diagnostic meetings. This centre reported discussing $60 \%$ of cases at their formal meetings.

\section{Meeting format, regularity and team composition}

In the 350 centres $(76.6 \%)$ holding formal meetings, these meetings were most often solely face-to-face $(\mathrm{n}=280(80.0 \%))$ and almost all $(\mathrm{n}=340(97.1 \%))$ had some face-to-face component. Formal meetings were most often held once a week ( $\mathrm{n}=122(34.9 \%))$, were $31-60 \mathrm{~min}$ long $(\mathrm{n}=178(50.9 \%))$, discussed $1-5$ cases $(\mathrm{n}=212(60.6 \%))$ and had a median (IQR) of $4(3-5)$ disciplines in attendance. Most meetings $\leqslant 90 \mathrm{~min}$ long discussed $1-5$ cases (186 out of 290 (64.1\%)) compared with 43.3\% (26 out of 60) of meetings $>90$ min long (figure 3).

The format of meetings varied regionally and by centre type (table 3). ILD academic centres were more likely to hold formal meetings (196 out of 205 (95.6\%)) than either non-ILD academic centres (87 out of 

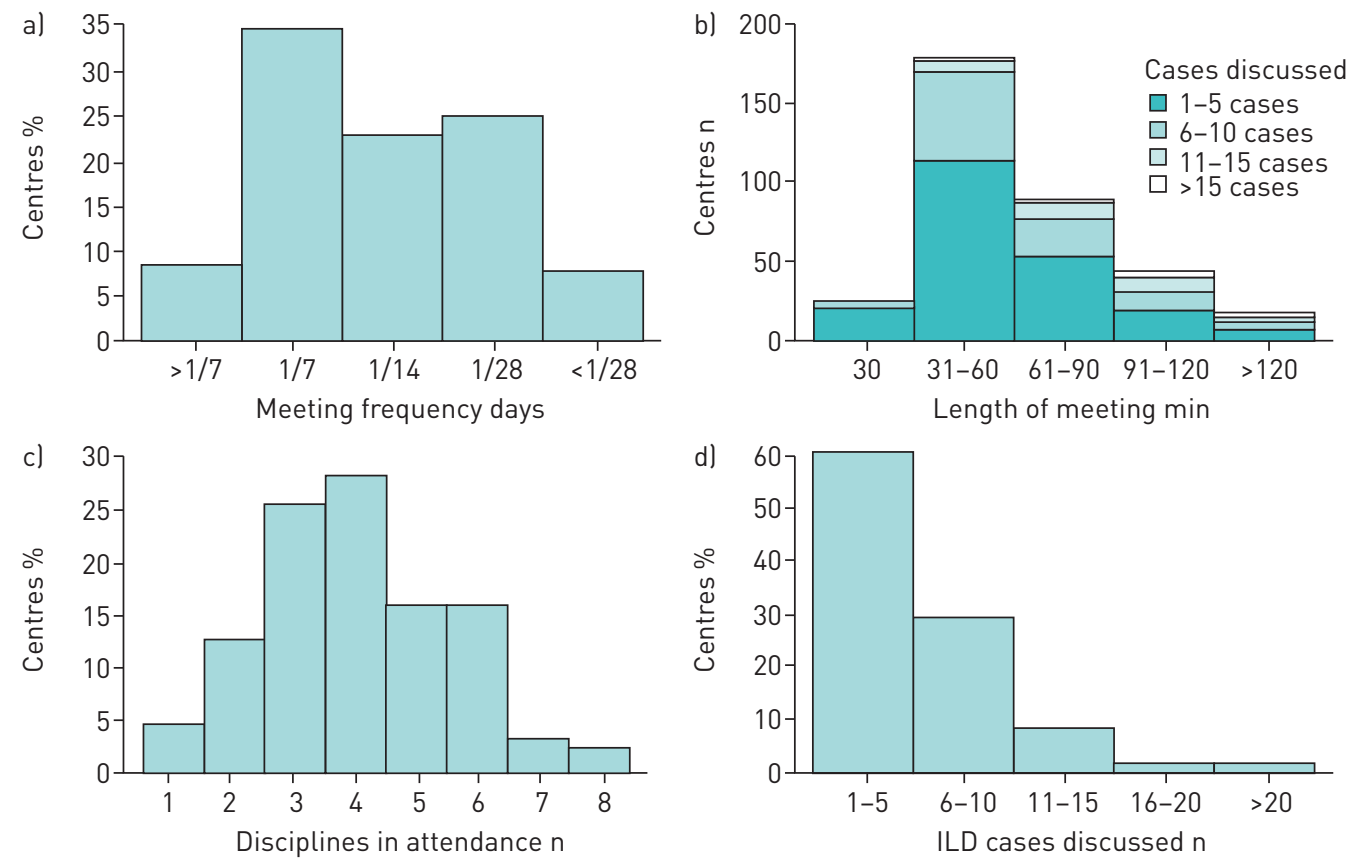

FIGURE 3 Key characteristics of formal meetings: al frequency, b) length by cases discussed, c) disciplines in attendance and d) interstitial lung disease cases discussed.

$133(65.4 \%)$ ) or non-academic centres (67 out of $119(56.3 \%)$; $\mathrm{p}<0.001)$, as were centres in high-income countries (250 out of $306(81.7 \%)$ ) compared with centres in other countries (100 out of $151(66.2 \%)$; $\mathrm{p}<0.001$ ). ILD academic centres were more likely to have meetings at least once every 2 weeks (147 out of $196(75.0 \%))$ compared with non-ILD academic centres (51 out of $87(58.6 \%)$ ) and non-academic centres (36 out of 67 (53.7\%); $\mathrm{p}=0.001$ ).

Most centres reported having pulmonology $(n=349(99.7 \%))$ and radiology $(n=320(91.4 \%))$ specialists in attendance; a lower proportion reported having histopathology $(n=232(66.3 \%))$ and rheumatology $(n=130$

\section{TABLE 3 Meeting characteristics by centre type, country income and region}

\begin{tabular}{|c|c|c|c|c|c|}
\hline & $\begin{array}{l}\text { Hold formal } \\
\text { meetings for } \\
\text { ILD cases }\end{array}$ & $\begin{array}{l}\text { Hold meetings } \\
\text { every } 2 \text { weeks } \\
\text { or more }\end{array}$ & $\begin{array}{c}\text { Hold meetings } \\
\text { solely } \\
\text { face-to-face }\end{array}$ & $\begin{array}{l}\text { Hold meetings } \\
\text { of } 31-60 \mathrm{~min} \\
\text { duration }\end{array}$ & $\begin{array}{l}\text { Have at least } \\
\text { four disciplines } \\
\text { in attendance }\end{array}$ \\
\hline \multicolumn{6}{|l|}{ Centre type } \\
\hline ILD academic & $196(95.6)$ & $147(75.0)$ & $156(79.6)$ & 97 (49.5) & $129(65.8)$ \\
\hline $\begin{array}{l}\text { Non-ILD } \\
\quad \text { academic }\end{array}$ & $87(65.4)$ & $51(58.6)$ & 78 (89.7) & 49 (56.3) & $44(50.6)$ \\
\hline Non-academic & 67 (56.3) & 36 (53.7) & 46 (68.7) & 32 (47.8) & $29(43.4)$ \\
\hline \multicolumn{6}{|l|}{ Country income } \\
\hline High & $250(81.7)$ & $167(66.8)$ & 209 (83.6) & $131(52.4)$ & $152(60.8)$ \\
\hline Upper-middle & 84 (68.9) & $58(69.0)$ & 59 (70.2) & 42 (50.0) & 44 (52.4) \\
\hline Lower-middle & 15 (55.5) & 8 (53.3) & 11 (73.3) & 5 (33.3) & 5 (33.3) \\
\hline Low & $1(50.0)$ & $1(100.0)$ & $1(100.0)$ & $0(0.0)$ & $1(100.0)$ \\
\hline \multicolumn{6}{|l|}{ Region } \\
\hline Africa & 8 (50.0) & 5 (62.5) & $8(100.0)$ & $6(75.0)$ & 3 (37.5) \\
\hline Asia-Pacific & 95 (79.2) & $67(70.5)$ & 71 (74.7) & $45(47.4)$ & $46(48.4)$ \\
\hline Europe & 148 (85.5) & $90(60.8)$ & $120(81.1)$ & $72(48.6)$ & 92 (62.2) \\
\hline Middle East & 10 (41.7) & 5 (50.0) & $9(90.0)$ & $5(50.0)$ & $5(50.0)$ \\
\hline North America & $47(74.6)$ & 34 (78.7) & 41 (87.2) & 30 (63.8) & $32(68.1)$ \\
\hline $\begin{array}{l}\text { South and Latin } \\
\text { America }\end{array}$ & 42 (68.9) & $33(78.6)$ & $31(73.8)$ & $20(47.6)$ & $24(57.1)$ \\
\hline All centres & $350(76.6)$ & 234 (66.9) & $280(80.0)$ & 178 (50.9) & 202 (57.7) \\
\hline
\end{tabular}



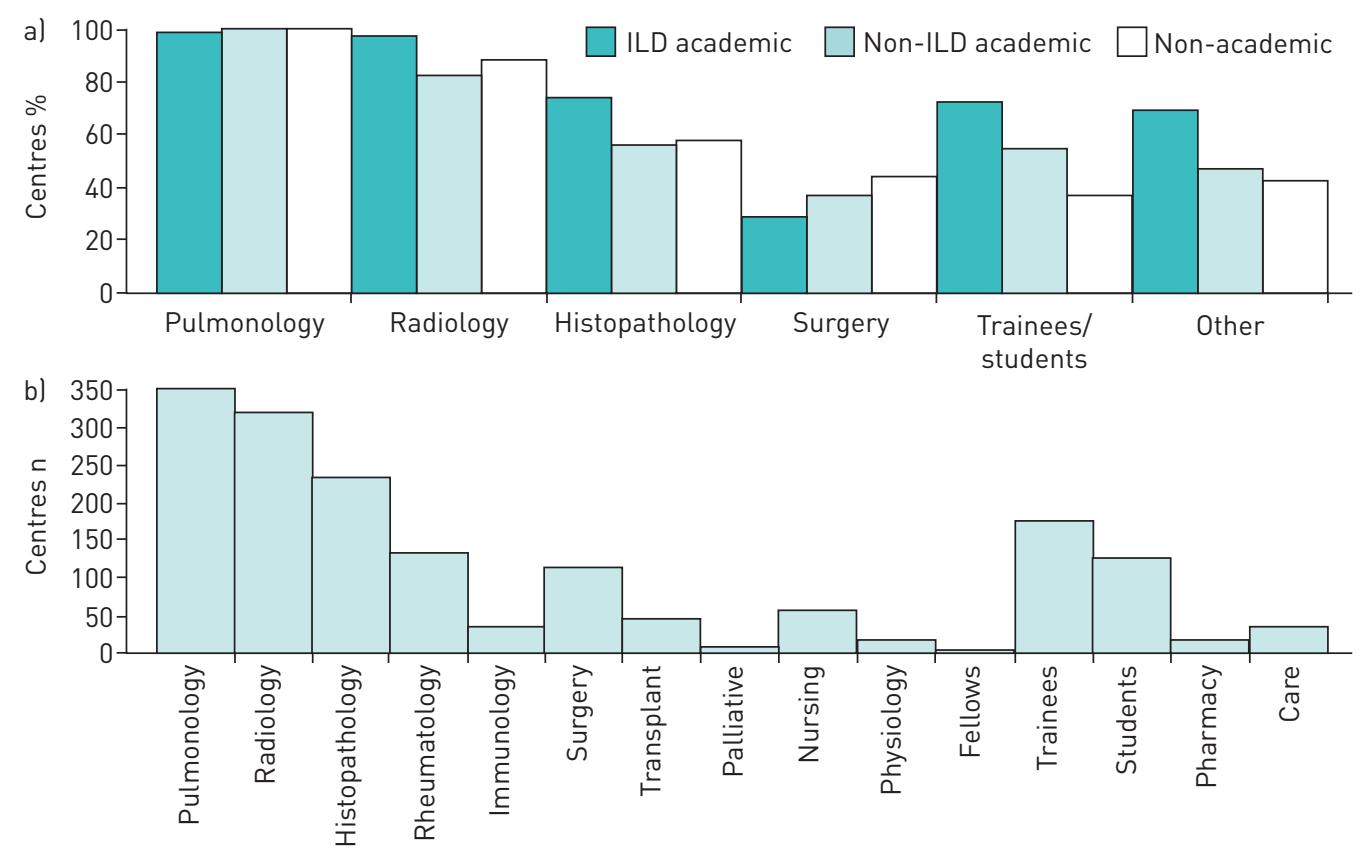

FIGURE 4 Disciplines and trainees regularly attending formal meetings by a) centre type and b) number of centres ( $n=350$ ). ILD: interstitial lung disease.

(37.1\%)) specialists in attendance (figure 4). ILD academic centres were more likely to have histopathology specialists $(\mathrm{n}=145(74.0 \%))$ in attendance than non-ILD academic centres $(\mathrm{n}=49(56.3 \%))$ or non-academic centres $(\mathrm{n}=38$ (56.7\%); $\mathrm{p}=0.003$ ) (figure 4) and were also more likely to have rheumatology specialists $(\mathrm{n}=83(42.3 \%))$ in attendance than other centres $(\mathrm{n}=30(34.5 \%)$ and $\mathrm{n}=17(25.4 \%)$, respectively; $\mathrm{p}=0.039)$.

\section{Meeting scope}

Most centres discussed diagnosis $(\mathrm{n}=346(98.9 \%))$, available diagnostic evidence $(\mathrm{n}=339(96.9 \%))$, recommended diagnostic tests $(\mathrm{n}=334(95.4 \%))$ and available therapeutic treatment $(\mathrm{n}=332(94.9 \%))$. Prognosis and supportive care were discussed by $86.0 \%(\mathrm{n}=301)$ and $66.9 \%(\mathrm{n}=234)$, respectively.

\section{Access to antifibrotic agents}

Antifibrotic agents (nintedanib or pirfenidone) were available to 372 of the 457 centres (81.4\%), among which $118(31.7 \%)$ required the permission of an MDT for access. A greater proportion of ILD academic centres had access to antifibrotics (180 out of 205 (87.8\%)) than non-ILD academic centres (100 out of $133(75.2 \%)$ ) or non-academic centres (92 out of 119 (77.3\%); p=0.006). However, ILD academic centres were more likely to require MDT permission to access them (69 out of $180(38.3 \%)$ ) than either non-ILD academic centres (20 out of $100(20.0 \%)$ ) or non-academic centres (29 out of $92(31.5 \%)$; $\mathrm{p}=0.007)$. Of the 306 centres in high-income countries, $89.5 \%(\mathrm{n}=274)$ had access to antifibrotics, compared with $64.8 \%(79$ out of 122) in upper-middle-income countries and 70.4\% (19 out of 27) in lower-middle-income countries $(\mathrm{p}<0.001)$. Neither of the two centres in low-income countries had access to antifibrotics and only $25 \%$ (four out of 12) of centres in Africa reported access, compared with almost all (62 out of 63 (98.4\%)) centres in North America. In contrast to centres in North America (three out of $63(4.8 \%)$ ) and Africa (none out of four $(0.0 \%)$ ), which rarely reported needing MDT permission to access antifibrotics, $45.6 \%$ (67 out of 147) of centres in Europe required MDT permission.

\section{BRIC countries}

Reponses from BRIC countries (i.e. Brazil $(n=10)$, Russia $(n=25)$, India $(n=19)$ and China $(n=9))$ accounted for $13.8 \%$ of all responses. Responses from BRIC countries were more often from academic ILD centres (32 out of $963(50.8 \%)$ ) than non-BRIC countries (173 out of $394(43.9 \%)$ ), although India had more non-academic centres $(10(52.6 \%))$ than any other type. The BRIC countries discussed new cases in an MDT meeting $(n=53(84.1 \%))$ more often than non-BRIC countries $(n=309(78.4 \%))$, although fewer meetings were formal (median 50\% compared with $80 \%)$ ). $50 \%(\mathrm{n}=5)$ of centres reported access to antifibrotics in Brazil, 56\% ( $\mathrm{n}=14)$ of centres in Russia, and $100 \%$ of centres in India $(\mathrm{n}=19)$ and China $(\mathrm{n}=9)$. 


\section{Discussion}

Knowledge of ILD diagnostic practices outside of well-characterised specialist centres is limited, particularly those used within the BRIC nations that contribute almost half of the world's population and in lower-income settings. This inclusive, descriptive study is a first attempt to look beyond well-resourced ILD centres and to understand diagnostic practice on a more global scale: across a range of countries, income and resource settings. Although participating centres were ostensibly located in high-income $(67 \%)$ or middle-income (32.6\%) countries, only $45 \%$ were self-described as academic ILD centres and there was participation (at some level) from all global regions: Europe contributed 37.9\% of centres, Asia-Pacific 26.3\%, North America 13.8\%, South and Latin America 13.3\%, Middle East 5.3\%, and Africa 3.5\%.

Overall, almost $80 \%$ of the centres report discussing new ILD cases and referrals at MDT meetings (ranging from $97 \%$ of academic ILD centres to $60 \%$ of non-academic centres). Although not designed to be representative of the global ILD community, this broad respondent group suggests the MDT approach to ILD diagnosis is widely adopted in routine care, across all participating countries, income and care settings. While most centres appear to conduct face-to-face meetings, which could be assumed to facilitate easier information exchange, the use of video conferencing and other remote communication aids may be integral to accessing critical ILD expertise in remote and underresourced settings.

The structure of the MDT meetings described by participating centres was broadly similar, but a "minimum MDT standard" remains hard to define. While some MDT meeting characteristics (e.g. length and number of cases discussed) are likely to be driven by the number of cases seen by the centre, others (e.g. range of specialities in attendance and diagnostic tests performed) may be influenced by available resources or expertise. Consistent with this, speciality representation varied between centres. While pulmonology was almost always represented (99.7\% of centres), and radiology at most centres (91.4\%), histopathology specialist attendance was less frequent, with approximately a third of centres (including $26 \%$ of academic ILD centres) having no pathology attendee. It is possible this is driven by a low proportion of cases requiring biopsies to establish diagnosis. However, given the crucial role of histopathologists in lung biopsy interpretation and the inclusion of pathology attendance as a defining factor of an MDT in two recent guidelines [6, 11], the reasons for this merit investigation. Further research to determine the effect that such differences in MDT composition and practice have on the diagnosis of ILD and subsequent outcomes is required, and is planned as a second phase of this research.

The lack of data from centres in low-income countries and uneven geographic distribution of responding centres are key limitations of this study. While the multiple recruitment channels used ensured the participant group was broad and inclusive, the self-selecting nature of respondents means it is unlikely to be representative and may underestimate the true extent of ILD diagnostic heterogeneity.

Despite these limitations, the concept of the MDT appears to be embodied in real-world diagnostic practice, with most centres, irrespective of global region, economic status, academic or ILD specialism, conducting face-to-face formal meetings involving representatives of multiple specialities to discuss ILD case diagnosis and management. This finding will inform the appropriate design of future diagnostic agreements studies required to guide optimal diagnostic pathway recommendations. It may also be of interest to those involved in the design and delivery of trial programmes for emerging ILD therapeutics where recruitment efforts have traditionally been focused on a relatively small number of recognised ILD centres whose pooled caseloads may struggle to meet growing enrolment demands.

Acknowledgements: The authors thank all 570 responding centres (supplementary file S3) for their time and contribution to expanding the understanding of diagnostic practice globally. We also thank the study's national and regional leads and steering committee, members of the Respiratory Effectiveness Group (REG) IPF/ILD working group, who participated in discussions and reviewed the study report, and Anjan Nibber (University of Oxford Medical School, Oxford, UK) who performed interim analyses.

REG IPF/ILD Working Group collaborators: Aileen David-Wang (University of the Philippines College of Medicine and Philippine General Hospital, Manila, Philippines), Antonio Morais (Centro Hospitalar São João, Oporto, Portugal), Arata Azuma (Nippon Medical School, Tokyo, Japan), Bruno Crestani (Université Paris Diderot, Paris, France), Carlo Vancheri (University of Catania, Catania, Italy), Carole Youakim (Mount Lebanon Hospital, Beirut, Lebanon), Charlene D. Fell (University of Calgary, Calgary, AB, Canada), Christopher J. Ryerson (University of British Columbia Vancouver, BC, Canada), Demosthenes Bouros (National and Kapodistrian University of Athens, Athens, Greece), Elisabeth Bendstrup (Aarhus University Hospital, Aarhus, Denmark), Ferran Morell (Vall d'Hebron Institut of Research (VHIR) and Pulmonology Service, Barcelona, Spain), Francesco Bonella (University of Duisburg-Essen, Essen, Germany), Ganesh Raghu (University of Washington, Seattle, WA, USA), George Christoff (Medical University Sofia, Sofia, Bulgaria), Giovanni Ferrara (Karolinska University Hospital, Stockholm, Sweden), Ian Glaspole (Alfred Health, Melbourne, Australia), Ivan Rosas (Brigham and Women's Hospital, Harvard Medical School, Boston, MA, USA), Jürgen Behr (University of Munich and Asklepios Fachkliniken Gauting, Munich, Germany), Kaissa DeBoer (University of Ottowa, Ottowa, ON, Canada), Katerina M. Antoniou (University of Crete, Heraklion, Greece), Keertan Dheda (University of Cape Town and Groote Schuur Hospital, Cape Town, South Africa), Kevin Brown (National Jewish Health, Denver, CO, USA), Lurdes Planas-Cerezales (University Hospital of Bellvitge, Barcelona, Spain), Magnus Sköld 
(Karolinska University Hospital), Manuela Funke (University Hospital Bern, Bern, Switzerland), Maria Molina-Molina (University Hospital of Bellvitge), Mariano Mazzei (University of Buenos Aires, Buenos Aires, Argentina), Martin Kolb (McMaster University, Hamilton, ON, Canada), Moises Selman (Instituto Nacional de Enfermedades Respiratorias "Ismael Cosio Villegas", Mexico City, Mexico), Paola Rottoli (University of Siena, Siena, Italy), Paolo Spagnolo (University of Padova, Padova, Italy), Pilar Rivera-Ortega (Manchester University NHS Trust, Manchester, UK), Sergey Avdeev (Sechenov First Moscow State Medical University, Moscow, Russia), Silvia Quadrelli (Sanatorio Güemes University Hospital, Buenos Aires, Argentina), Tamera J. Corte (Royal Prince Alfred Hospital and University of Sydney, Sydney, Australia), Toby M. Maher (Royal Brompton Hospital and Imperial College London, London, UK), Vincent Cottin (Hopital L. Pradel, Lyon, France), Wim Wuyts (University Hospitals Leuven, Leuven, Belgium) and Zuo Jun Xu (Peking Union Medical College Hospital, Beijing, China).

Conflict of interest: L. Richeldi reports personal fees for consulting activity from Sanofi-Aventis, ImmuneWorks, Celgene, Nitto, Bristol-Myers Squibb and Pliant Therapeutics, personal fees for membership of an advisory board from Roche, Fibrogen and Promedior, speaker fees from Shionogi, grants and personal fees for membership of a steering committee from Boehringer Ingelheim, and personal fees for editorial activity from DynaMed, outside the submitted work. N. Launders reports that the Respiratory Effectiveness Group received grants from Roche, Boehringer Ingelheim and Three Lakes Partners, and nonfinancial support from Veracyte, during the conduct of the study. F. Martinez reports personal fees, nonfinancial support and other support from Boehringer Ingelheim during the conduct of the study; personal fees and nonfinancial support from the American College of Chest Physicians, AstraZeneca, Boehringer Ingelheim, Continuing Education, ConCert, Genentech, GlaxoSmithKline, Miller Communications, the National Association for Continuing Education, Novartis, Pearl Pharmaceuticals, PeerView Communications, Prime Communications, the Puerto Rican Respiratory Society, Chiesi, Roche, Sunovion, Theravance, Potomac, the University of Alabama Birmingham, the Physicians Education Resource, the Canadian Respiratory Network and Teva, nonfinancial support from ProterrixBio, the Inova Fairfax Health System, Gilead, Nitto, Patara and Zambon, personal fees from Columbia University, Integritas, MD Magazine, Methodist Hospital Brooklyn, New York University, Unity, UpToDate, WebMD/MedScape, the Western Connecticut Health Network, Academic CME, PlatformIQ, the American Thoracic Society and Rockpointe, other support from Afferent/Merck, Biogen, Veracyte, Prometic, Bayer and Bridge Biotherapeutics, and grants from the NIH, Rare Disease Healthcare Communications and ProMedior, outside the submitted work. S.L.F. Walsh has nothing to disclose. J. Myers has nothing to disclose. B. Wang has nothing to disclose. M. Jones has nothing to disclose. A. Chisholm reports she worked for the Respiratory Effectiveness Group during the design and data capture stages of the study; she now works for Syneos Health. She has no conflicts of interest to declare in relation to this study. K.R. Flaherty reports grants and personal fees from Boehringer Ingelheim and Roche/ Genentech, personal fees from Veracyte, Aeolus, Pharmakea, Sanofi-Genzyme and Fibrogen, outside the submitted work.

Support statement: This REG (http://effectivenessevaluation.org) study was supported by grants from Roche, Boehringer Ingelheim and Three Lakes Partners, and by in-kind support from Veracyte. Funding information for this article has been deposited with the Crossref Funder Registry.

\section{References}

1 Hutchinson J, Fogarty A, Hubbard R, et al. Global incidence and mortality of idiopathic pulmonary fibrosis: a systematic review. Eur Respir J 2015; 46: 795-806.

2 Ley B, Collard HR. Epidemiology of idiopathic pulmonary fibrosis. Clin Epidemiol 2013; 5: 483-492.

3 Fleetwood K, McCool R, Glanville J, et al. Systematic review and network meta-analysis of idiopathic pulmonary fibrosis treatments. J Manag Care Spec Pharm 2017; 23: 3-b Suppl., S5-S16.

4 American Thoracic Society, European Respiratory Society. American Thoracic Society/European Respiratory Society international multidisciplinary consensus classification of the idiopathic interstitial pneumonias. Am J Respir Crit Care Med 2002; 165: 277-304.

5 Travis WD, Costabel U, Hansell DM, et al. An official American Thoracic Society/European Respiratory Society statement: update of the international multidisciplinary classification of the idiopathic interstitial pneumonias. Am J Respir Crit Care Med 2013; 188: 733-748.

6 Raghu G, Remy-Jardin M, Myers JL, et al. Diagnosis of idiopathic pulmonary fibrosis. An official ATS/ERS/JRS/ ALAT clinical practice guideline. Am J Respir Crit Care Med 2018; 198: e44-e68.

7 Flaherty KR, King TE, Raghu G, et al. Idiopathic interstitial pneumonia: what is the effect of a multidisciplinary approach to diagnosis? Am J Respir Crit Care Med 2004; 170: 904-910.

8 Walsh SL, Wells AU, Desai SR, et al. Multicentre evaluation of multidisciplinary team meeting agreement on diagnosis in diffuse parenchymal lung disease: a case-cohort study. Lancet Respir Med 2016; 4: 557-565.

9 Walsh SLF, Maher TM, Kolb M, et al. Diagnostic accuracy of a clinical diagnosis of idiopathic pulmonary fibrosis: an international case-cohort study. Eur Respir J 2017; 50: 1700936.

10 De Sadeleer LJ, Meert C, Yserbyt J, et al. Diagnostic ability of a dynamic multidisciplinary discussion in interstitial lung diseases: a retrospective observational study of 938 cases. Chest 2018; 153: 1416-1423.

11 Lynch DA, Sverzellati N, Travis WD, et al. Diagnostic criteria for idiopathic pulmonary fibrosis: a Fleischner Society White Paper. Lancet Respir Med 2018; 6: 138-153.

12 Cottin V, Crestani B, Cadranel J, et al. French practical guidelines for the diagnosis and management of idiopathic pulmonary fibrosis - 2017 update. Full-length version. Rev Mal Respir 2017; 34: 900-968.

13 Belloli E, Rosenbluth M, Choi Y, et al. AB015. Current diagnostic approaches in ILD: ILD vs. non-specialty clinics. J Thorac Dis 2016; 8: Suppl. 5, AB015.

14 Jo HE, Corte TJ, Moodley Y, et al. Evaluating the interstitial lung disease multidisciplinary meeting: a survey of expert centres. BMC Pulm Med 2016; 16: 22.

15 World Bank. World Bank Country and Lending Groups. 2017. https://datahelpdesk.worldbank.org/knowledgebase/ articles/906519-world-bank-country-and-lending-groups Date last accessed: September 25, 2017. 